\title{
A GLOBAL EXISTENCE THEOREM FOR AUTONOMOUS DIFFERENTIAL EQUATIONS IN A BANACH SPACE
}

\author{
R. H. MARTIN, JR.
}

Abstract. Let $E$ be a Banach space and let $A$ be a continuous function from $E$ into $E$. Sufficient conditions are given to insure that the differential equation $u^{\prime}(t)=A u(t)$ has a unique solution on $[0, \infty)$ for each initial value in $E$. One consequence of this result is that if $-A$ is monotonic, then $-A$ is $m$-monotonic and $A$ is the generator of a nonexpansive semigroup of operators.

Let $E$ be a Banach space over the real or complex field and let | $\mid$ denote the norm on $E$. If $A$ is a continuous function from $E$ into $E$, we will give a sufficient condition for the autonomous differential equation

$$
u^{\prime}(t)=A u(t)
$$

to have a unique solution $u(\cdot, z)$ defined on $[0, \infty)$ such that $u(0, z)=z$ for each $z$ in $E$. One consequence of this result is that if $A$ is continuous and $-A$ is monotonic, then $-A$ is $m$-monotonic and $A$ is the generator of a nonexpansive semigroup of operators. The principal tool in this paper is the one-sided derivative of the norm on $E$ which will be used as a Liapunov function.

Definition 1. If $A$ is a function from $E$ into $E$ and $x$ and $y$ are in $E$, define

(i) $D_{+}[x, y, A]=\lim _{h \rightarrow+0}(|x-y+h[A x-A y]|-|x-y|) / h$ and

(ii) $D_{-}[x, y, A]=\lim _{h \rightarrow-0}(|x-y+h[A x-A y]|-|x-y|) / h$.

REMARK 1. Each of the above limits exists since the function $h \rightarrow|x-y+h[A x-A y]|$ is convex. Furthermore, $D_{-}[x, y, A]$ $\leqq D_{+}[x, y, A]$ for all $x$ and $y$ in $E$.

Example 1. Let $E^{*}$ denote the dual of $E$ and for each $x$ in $E$ let $G(x)$ denote the set of all $g$ in $E^{*}$ such that $(x, g)=|x|$ and $|g|=1$. In $\left[8\right.$, Corollary 2.2] it is shown that $D_{+}[x, y, A]$ $=\sup \{\operatorname{Re}(A x-A y, g): g$ is in $G(x-y)\}$. Consequently, $D_{-}[x, y, A]$ $=-D_{+}[x, y,-A]=\inf \{\operatorname{Re}(A x-A y, g): g$ is in $G(x-y)\}$. For each $x$ in $E$ let $F(x)$ denote the set of all $f$ in $E^{*}$ such that $(x, f)=|x|^{2}$ and $|f|=|x|$. Note that if $x \neq 0$ then $f$ is in $F(x)$ if and only if $f /|x|$ is in $G(x)$. Thus $-A$ is accretive on $E$ (i.e. $\operatorname{Re}(A x-A y, f) \leqq 0$

Received by the editors February 3, 1970.

AMS 1969 subject classifications. Primary 3495; Secondary 4750.

Key words and phrases. Banach space, one-sided derivative of the norm, autonomous differential equation, monotonic, semigroup of nonlinear operators. 
for all $x$ and $y$ in $E$ and all $f$ in $F(x-y)$ - see [1]) if and only if $D_{+}[x, y, A] \leqq 0$ for all $x$ and $y$ in $E$ and $-A$ is monotonic on $E$ (i.e. $\operatorname{Re}(A x-A y, f) \leqq 0$ for all $x$ and $y$ in $E$ and some $f$ in $F(x-y)$ see [4]) if and only if $D_{-}[x, y, A] \leqq 0$.

Let $R$ denote the space of real numbers and suppose that $\alpha$ is a function from $R$ into $R$ which satisfies each of the following conditions:

(C1) $\alpha$ is continuous, $\alpha(0)=0$, and $\phi(t, 0)=0$ for all $t$ in $[0, \infty)$ is the unique solution to $y^{\prime}=\alpha(y)$ such that $\phi(0,0)=0$.

(C2) For each $\rho>0$ the maximal solution $\phi(\cdot, \rho)$ to $y^{\prime}=\alpha(y)$ such that $\phi(0, \rho)=\rho$ exists on $[0, \infty)$.

LEMMA 1. Let $q$ be a function from the interval $(a, b)$ into $E$ and let $p(t)=|q(t)|$ for each $t$ in $(a, b)$.

(i) If $q_{+}^{\prime}(t)$ exists then $p_{+}^{\prime}(t)$ exists and

$$
p_{+}^{\prime}(t)=\lim _{h \rightarrow+0}\left(\left|q(t)+h q_{+}^{\prime}(t)\right|-|q(t)|\right) / h .
$$

(ii) If $q_{-}^{\prime}(t)$ exists then $p_{-}^{\prime}(t)$ exists and

$$
p_{-}^{\prime}(t)=\lim _{h \rightarrow-0}\left(\left|q(t)+h q_{-}^{\prime}(t)\right|-|q(t)|\right) / h .
$$

For a proof see $[3$, p. 3$]$.

LEMMA 2. Let $\alpha$ satisfy conditions (C1) and (C2), let $T$ be a positive number, and let $\left(\delta_{n}\right)_{1}^{\infty}$ and $\left(\rho_{n}\right)_{1}^{\infty}$ be sequences of nonnegative numbers such that $\lim _{n \rightarrow \infty} \delta_{n}=0$ and $\lim _{n \rightarrow \infty} \rho_{n}=0$. For each $n \geqq 1$ let $\psi_{n}$ be a solution to $y^{\prime}=\alpha(y)+\delta_{n}$ such that $\psi_{n}(0)=\rho_{n}$. Then for sufficiently large $n, \psi_{n}$ is defined on $[0, T]$ and $\lim _{n \rightarrow \infty} \psi_{n}(t)=0$ uniformly on $[0, T]$.

For a proof see $[3$, Theorem 3, p. 17].

LEMMA 3. Let $\beta$ be a continuous function from $R$ into $R$, let $\rho$ be in $R$, and let $r$ be the maximal solution to the differential equation $y^{\prime}=\beta(y)$ such that $r(0)=\rho$. If $r$ is defined on $[0, T]$ and $p$ is a continuous function from $[0, T]$ into $R$ such that $p(0) \leqq \rho$ and $p_{-}^{\prime}(t) \leqq \beta(p(t))$ for each $t$ in $(0, T]$, then $p(t) \leqq r(t)$ for all $t$ in $[0, T]$.

For a proof see [6, Theorem 1.4.1, p. 15].

THEOREM 1. Let $\alpha$ and $\phi$ satisfy the conditions (C1) and (C2) and suppose that $A$ is a function from $E$ into $E$ which satisfies each of the following:

(i) $A$ is continuous on $E$. 
(ii) $D_{-}[x, y, A] \leqq \alpha(|x-y|)$ for all $x$ and $y$ in $E$.

Then for each $z$ in $E$ there is a continuously differentiable function $u(\cdot, z)$ from $[0, \infty)$ into $E$ such that $u(0, z)=z$ and $u^{\prime}(t, z)=A u(t, z)$ for all $t$ in $[0, \infty)$. Furthermore, if $z$ and $w$ are in $E$, then $|u(t, z)-u(t, w)| \leqq \phi(t,|z-w|)$ for all t in $[0, \infty)$.

REMARK 2. Note that if $D_{+}[x, y, A] \leqq \alpha(|x-y|)$ for all $x$ and $y$ in $E$, then (ii) holds by Remark 1.

REMARK 3. Note that condition (ii) is equivalent to $\operatorname{Re}(A x-A y, g)$ $\leqq \alpha(|x-y|)$ for all $x$ and $y$ in $E$ and some $g$ in $G(x-y)$ (see Example 1).

Theorem 1 will be proved with a sequence of lemmas each of which is under the suppositions of Theorem 1 . Let $z$ be in $E$ and let $V$ be a neighborhood of $z$ for which there is a number $M$ such that $|A x| \leqq M$ for all $x$ in $V$. Furthermore, choose $T>0$ sufficiently small so that if $x$ is in $E$ with $|x-z| \leqq M T$, then $x$ is in $V$. Now let $\left(\epsilon_{n}\right)_{1}^{\infty}$ be a sequence of positive numbers such that $\lim _{n \rightarrow \infty} \epsilon_{n}=0$.

Lemma 4. For each positive integer $n$ there is a positive integer $N$ $=N(n)$, a partition $\left(t_{i}^{n}\right)_{i=0}^{N}$ of $[0, T]$, and a function $u_{n}$ from $[0, T]$ into $V$ such that:

(i) $\left|t_{i}^{n}-t_{i-0}^{n}\right| \leqq n^{-1}$ for each $1 \leqq i \leqq N$.

(ii) $\left|u_{n}(t)-u_{n}(s)\right| \leqq M|t-s|$ for all $t$ and $s$ in $[0, T]$.

(iii) If $t$ is in $\left(t_{i-1}^{n}, t_{i}^{n}\right]$, then $\left(u_{n}\right)_{-}^{\prime}(t)$ exists and equals $A u_{n}\left(t_{i-1}^{n}\right)$ for each $1 \leqq i \leqq n$.

(iv) If $\left|x-u_{n}\left(t_{i-1}^{n}\right)\right| \leqq M\left(t_{i}^{n}-t_{i-1}^{n}\right)$ then $\left|A x-A u_{n}\left(t_{i-1}^{n}\right)\right| \leqq \epsilon_{n}$.

Proof. Let $t_{0}^{n}=0$ and $u_{n}\left(t_{0}^{n}\right)=z$. Inductively, for each positive integer $i$ choose $\delta_{i}^{n}$ in $\left[0, n^{-1}\right]$ such that $t_{i-1}^{n}+\delta_{i}^{n} \leqq T$ and

(1) If $\left|x-u_{n}\left(t_{i-1}^{n}\right)\right| \leqq M \delta_{i}^{n}$ then $\left|A x-A u_{n}\left(t_{i-1}^{n}\right)\right| \leqq \epsilon_{n}$.

(2) Exactly one of the following holds:

(a) $\delta_{i}^{n}=n^{-1}$ or $t_{i-1}^{n}+\delta_{i}^{n}=T$.

(b) Alternative (a) does not hold and for each $\beta>0$ there is an $x_{\beta}$ in $E$ such that $M \delta_{i}^{n}<\left|x_{\beta}-u_{n}\left(t_{i-1}^{n}\right)\right| \leqq M \delta_{i}^{n}+\beta$ and $\left|A x_{\beta}-A u_{n}\left(t_{i-1}^{n}\right)\right|$ $>\epsilon_{n}$.

Take $t_{i}^{n}=t_{i-1}^{n}+\delta_{i}^{n}$ and for each $t$ in $\left[t_{i-1}^{n}, t_{i}^{n}\right]$ define $u_{n}(t)=u_{n}\left(t_{i-1}^{n}\right)$ $+\left(t-t_{i-1}^{n}\right) A u_{n}\left(t_{i-1}^{n}\right)$. By the choice of $M$ and $T$ it is clear that if $t$ and $s$ are in $[0, t]_{i}^{n}$ for some positive integer $i$, then $\left|u_{n}(t)-u_{n}(s)\right|$ $\leqq M|t-s|$ and hence $\left|u_{n}(t)-z\right|=\left|u_{n}(t)-u_{n}(0)\right| \leqq M t \leqq M T$ so that $u_{n}(t)$ is in $V$. The claim is that there is an integer $N$ such that $t_{N}^{n}=T$. Suppose, for contradiction, that this is not the case. Since $t_{i-1}^{n}<t_{t}^{n}<T$ for all $i \geqq 1$, there is an $s_{0}$ in $(0, T]$ such that $\lim _{i \rightarrow \infty} t_{i}^{n}=s_{0}$. Since 
$\left|u_{n}\left(t_{i}^{n}\right)-u_{n}\left(t_{j}^{n}\right)\right| \leqq M\left|t_{i}^{n}-t_{j}^{n}\right|$, the sequence $\left(u_{n}\left(t_{i}^{n}\right)\right)_{i=0}^{\infty}$ is Cauchy so let $x_{0}=\lim _{i \rightarrow \infty} u_{n}\left(t_{i}^{n}\right)$. Let $0<\rho<n^{-1}$ be such that if $\left|x-x_{0}\right| \leqq \rho$, then $\left|A x-A x_{0}\right| \leqq \epsilon_{n} / 3$. Choose an integer $k$ sufficiently large so that $\left|t_{k}^{n}-t_{k-1}^{n}\right|<\rho /(3 M+1)$ and $\left|u_{n}\left(t_{k-1}^{n}\right)-x_{0}\right| \leqq \rho / 3$. Since $\left|t_{k}^{n}-t_{k-1}^{n}\right|$ $<n^{-1}$ and $t_{k-1}^{n}<t_{k}^{n}<T$, alternative (b) of (2) must hold. However, by the choice of $k, x_{0}$, and $\rho$, if $x$ is in $E$ and $M \delta_{k}^{n}<\left|x-u_{n}\left(t_{k-1}^{n}\right)\right| \leqq M \delta_{k}^{n}$ $+\rho / 3$, then $\left|x-u_{n}\left(t_{k-1}^{n}\right)\right| \leqq M\left(t_{k}^{n}-t_{k-1}^{n}\right)+\rho / 3 \leqq 2 \rho / 3$ and hence

$$
\left|x-x_{0}\right| \leqq\left|x-u_{n}\left(t_{k-1}^{n}\right)\right|+\left|u_{n}\left(t_{k-1}^{n}\right)-x_{0}\right| \leqq 2 \rho / 3+\rho / 3=\rho
$$

so that

$$
\begin{aligned}
\left|A x-A u_{n}\left(t_{k-1}^{n}\right)\right| & \leqq\left|A x-A x_{0}\right|+\left|A x_{0}-A u_{n}\left(t_{k-1}^{n}\right)\right| \\
& \leqq \epsilon_{n} / 3+\epsilon_{n} / 3 \\
& <\epsilon_{n} .
\end{aligned}
$$

This is a contradiction to alternative (b) of (2). This contradiction shows that there exists such an integer $N$. The other assertions of the lemma now follow easily from the construction of $u_{n}$.

LEMMA 5. The sequence of functions $\left(u_{n}\right)_{0}^{\infty}$ converge uniformly to a function $u$ from $[0, T]$ into $V$ such that $u(0)=z$.

Proof. Using the notations of Lemma 4 , let $n$ and $m$ be positive integers and for each $t$ in $[0, T]$ define $p(t)=\left|u_{n}(t)-u_{m}(t)\right|$. Let $t$ be in $(0, T]$ and let $i$ and $j$ be integers such that $t$ is in $\left(t_{i-1}^{n}, t_{i}^{n}\right]$ and $t$ is in $\left(t_{j-1}^{m}, t_{j}^{m}\right]$. By Lemma 1 and part (iii) of Lemma 4 ,

$$
\begin{aligned}
p_{-}^{\prime}(t)= & \lim _{n \rightarrow-0}\left(\left|u_{n}(t)-u_{m}(t)+h\left[A u_{n}\left(t_{i-1}^{n}\right)-A u_{m}\left(t_{j-1}^{m}\right)\right]\right|\right. \\
\leqq & \left.-\left|u_{n}(t)-u_{m}(t)\right|\right) / h \\
\lim _{n \rightarrow-0}\left(\left|u_{n}(t)-u_{m}(t)+h\left[A u_{n}(t)-A u_{m}(t)\right]\right|\right. & \left.-\left|u_{n}(t)-u_{m}(t)\right|\right) / h \\
& +\left|A u_{n}\left(t_{i-1}^{n}\right)-A u_{n}(t)\right|+\left|A u_{m}(t)-A u_{m}\left(t_{j-1}^{m}\right)\right| \\
= & D_{-}\left[u_{n}(t), u_{m}(t), A\right]+\left|A u_{n}\left(t_{i-1}^{n}\right)-A u_{n}(t)\right| \\
& +\left|A u_{m}(t)-A u_{m}\left(t_{j-1}^{m}\right)\right| .
\end{aligned}
$$

By part (ii) of Lemma $4,\left|u_{n}\left(t_{i-1}^{n}\right)-u_{n}(t)\right| \leqq M\left|t-t_{i-1}^{n}\right| \leqq M\left|t_{i}^{n}-t_{i-1}^{n}\right|$ and so by part (iv) of Lemma $4,\left|A u_{n}\left(t_{i-1}^{n}\right)-A u_{n}(t)\right| \leqq \epsilon_{n}$. Analo- 
gously, $\left|A u_{m}(t)-A u_{m}\left(t_{j-1}^{m}\right)\right| \leqq \epsilon_{m}$. Thus, from supposition (ii) of Theorem 1,

$$
p^{\prime}-(t) \leqq \alpha(p(t))+\epsilon_{n}+\epsilon_{m}
$$

for each $t$ in $(0, T]$. Since $p(0)=0$ we have by Lemma 3 that $p(t)$ $\leqq \psi(t)$ where $\psi$ is the maximal solution to $y^{\prime}=\alpha(y)+\epsilon_{n}+\epsilon_{m}$ such that $\psi(0)=0$. It now follows from Lemma 2 that the sequence $\left(u_{n}(t)\right)_{1}^{\infty}$ is uniformly Cauchy on $[0, T]$ and hence converges uniformly on $[0, T]$ to a continuous function $u$ on $[0, T]$. Since $\left|u_{n}(t)-u_{n}(s)\right|$ $\leqq M|t-s|$ for all $n \geqq 1$, it is immediate that $|u(t)-u(s)| \leqq M|t-s|$ for all $t$ and $s$ in $[0, T]$. Since $u_{n}(0)=z$ for all $n \geqq 1, u(0)=z$ and hence $|u(t)-z| \leqq M t \leqq M T$ so that $u(t)$ is in $V$ for all $t$ in $[0, T]$. This completes the proof of the lemma.

LemMA 6. If $u$ is as in Lemma 5 , then $u(t)=z+\int_{0}^{t} A u(s) d s$ for all $t$ in $[0, T]$.

Proof. For each positive integer $n$ define the function $v_{n}$ from $[0, T]$ into $E$ by $v_{n}(t)=A u_{n}\left(t_{i-1}^{n}\right)$ whenever $t$ is in $\left(t_{i-1}^{n}, t_{i}^{n}\right]$ and $v_{n}(0)$ $=A z$. By the construction of $u_{n}$ in Lemma 4 we have that $\left|v_{n}(t)\right| \leqq M$ and

$$
u_{n}(t)=z+\int_{0}^{t} v_{n}(s) d s
$$

for all $t$ in $[0, T]$. If $t$ is in $\left(t_{i-1}^{n}, t_{i}^{n}\right]$, we have by parts (i) and (ii) of Lemma 4 that

$$
\begin{aligned}
\left|u(t)-u_{n}\left(t_{i-1}^{n}\right)\right| & \leqq\left|u(t)-u_{n}(t)\right|+\left|u_{n}(t)-u_{n}\left(t_{i-1}^{n}\right)\right| \\
& \leqq\left|u(t)-u_{n}(t)\right|+n^{-1} M .
\end{aligned}
$$

Thus, by Lemma 5 and the continuity of $A, \lim _{n \rightarrow \infty} v_{n}(t)=A u(t)$ for each $t$ in $[0, T]$. Since the sequence $\left(v_{n}\right)_{1}^{\infty}$ is uniformly bounded, it follows by bounded convergence that

$$
u(t)=\lim _{n \rightarrow \infty} u_{n}(t)=\lim _{n \rightarrow \infty} z+\int_{0}^{t} v_{n}(s) d s=z+\int_{0}^{t} A u(s) d s
$$

and the proof of the lemma is complete.

Lemma 6 shows that (ADE) has a local solution with initial value $z$ for each $z$ in $E$. We will now use this to show that each solution to $(\mathrm{ADE})$ can be defined on $[0, \infty)$.

Lemma 7. For each $z$ in $E$ there is a function $u(\cdot, z)$ from $[0, \infty)$ into $E$ such that $u(0, z)=z$ and $u(\cdot, z)$ is a solution to (ADE) on $[0, \infty)$, 
Proof. By Lemma 6 there is a $T>0$ such that $u(t, z)$ is defined for all $t$ in $[0, T)$. If $T<\infty$, let $0<h<T$ and for each $t$ in $[0, T-h)$ define $p(t)=|u(t+h, z)-u(t, z)|$. By Lemma $1, p_{-}^{\prime}(t)$ $=D_{-}[u(t+h), u(t), A]$ so by supposition (ii) of Theorem $1, p_{-}^{\prime}(t)$ $\leqq \alpha(p(t))$ for all $t$ in $(0, T-h)$. Thus, from Lemma 3,

$$
|u(t+h, z)-u(t, z)| \leqq \phi(t,|u(h, z)-u(0, z)|)
$$

for all $t$ in $[0, T-h)$. Let $t+h$ and $t$ tend to $T$ from below, then $h$ must tend to 0 from above and by Lemma $2, \phi(t, \rho)$ tends to 0 as $\rho$ tends to +0 , uniformly for $t$ in a bounded subinterval of $[0, \infty)$. Consequently, $\lim _{h \rightarrow+0} \phi(t,|u(h, z)-u(0, z)|)=0$ uniformly for $t$ in $[0, T]$ and it follows that $u(t)$ tends to a limit as $t$ tends to $T$ from below. By Lemma $6 u(t, z)$ can be continued past $T$ and it follows that $u(t, z)$ can be defined for all $t$ in $[0, \infty)$ and the proof of the lemma is complete.

LemMa 8. If $z$ and $w$ are in $E$ and $t$ is in $[0, \infty)$, then

$$
|u(t, z)-u(t, w)| \leqq \phi(t,|z-w|) .
$$

Proof. For each $t$ in $[0, \infty)$ define $p(t)=|u(t, z)-u(t, w)|$. Then by Lemma 1 and supposition (ii) of Theorem 1 ,

$$
p_{-}^{\prime}(t)=D_{-}[u(t, z), u(t, w), A] \leqq \alpha(p(t))
$$

for all $t$ in $[0, \infty)$ and the assertion of the lemma follows easily from Lemma 3.

Since $\phi(t, 0)=0$ for all $t$ in $[0, \infty)$ the uniqueness of $u(\cdot, z)$ is immediate from Lemma 7 and the proof of Theorem 1 is complete.

Example 2. Suppose that $A$ is continuous and $-A$ is monotonic on $E$ (see Example 1). Then $D_{-}[x, y, A] \leqq 0$ for all $x$ and $y$ in $E$ so that if $\alpha(r)=0$ for all $r$ in $R$ (and hence $\phi(t, \rho)=\rho$ for all $t$ and $\rho$ in $[0, \infty)$ ), then each of the conditions of Theorem 1 are fulfilled. In particular, $A$ generates a semigroup of nonlinear operators on $E$. In [9] G. F. Webb proves this with the additional assumption that the image of $E$ under $I-\epsilon A$ (where $I$ is the identity function on $E$ ) is $E$ for each $\epsilon>0$ (i.e. $-A$ is $m$-monotonic on $E$ ). Webb also gives a product integral representation of the solution $u(t, z)$. However, if for each $y$ in $E, A-I-y$ generates a semigroup of nonlinear operators, then $-A$ is necessarily $m$-monotonic on $E$ (see [1, Theorem 3]). Since $A-I-y$ satisfies each of the suppositions of $A$ in Theorem 1, with $\alpha(\gamma)=-\gamma$, we have as a corollary to Theorem 1 that if $-A$ is continuous and monotonic, then $-A$ is $m$-monotonic. 
Remark 4. Suppose that $A$ satisfies the suppositions of Theorem 1 and let $x$ and $y$ be in $E$. If $g$ is in $G(x-y)$ (see Example 1) then $|g|=1$ and $(x-y, g)=|x-y|$ so that

$$
\begin{aligned}
\operatorname{Re}(A x-A y, g) & =\operatorname{Re}\left(\lim _{h \rightarrow+0}[u(h, x)-x-u(h, y)+y \mid / h, g)\right. \\
& =\lim _{h \rightarrow+0}[\operatorname{Re}(u(h, x)-u(h, y), g)-(x-y, g)] / h \\
& \leqq \lim _{h \rightarrow+0}[|u(h, x)-u(h, y)|-|x-y|] / h \\
& \leqq \lim _{h \rightarrow+0}[\phi(h,|x-y|)-\phi(0,|x-y|)] / h \\
& =\alpha(|x-y|) .
\end{aligned}
$$

Since this is true for each $g$ in $G(x-y)$ we have by Example 1 that $D_{+}[x, y, A] \leqq \alpha(|x-y|)$ for all $x$ and $y$ in $E$. In particular, if $-A$ is continuous and monotonic, then $-A$ is accretive.

REMARK 5. In [5], Kato shows that the conclusions of Theorem 1 are valid in the case that $\alpha(r)=0$ for each number $r$ and the space $E^{*}$ is uniformly convex. If $E$ is a general Banach space, $\alpha(r)=0$ for each number $r$, and the function $A$ is locally uniformly continuous on $E$, then Browder [2] shows that the conclusions of Theorem 1 are valid.

REMARK 6. In [7], the author proves a theorem concerning the existence and stability of critical points of the equation (ADE) in which local existence of solutions to (ADE) is assumed. In view of Theorem 1, one sees that this assumption is now redundant. In particular, Theorem 1 and methods similar to those used in [1] and [7] provide some techniques which can be used to establish fixed point theorems for operators in a Banach space.

\section{REFERENCES}

1. F. E. Browder, Nonlinear equations of evolution and nonlinear accretive operators in Banach spaces, Bull. Amer. Math. Soc. 73 (1967), 867-874. MR 38 \#580

2. - Nonlinear operators and nonlinear equations of evolution in Banach spaces, Proc. Sympos. Pure Math., vol. 18, Part 2, Amer. Math. Soc., Providence, R. I. (to appear).

3. W. A. Coppel, Stability and asymptotic behavior of differential equations, D. C. Heath, Boston, 1965. MR 32 \#7875.

4. T. Kato, Nonlinear semigroups and evolution equations, J. Math. Soc. Japan 19 (1967), 508-520. MR 37 \#1820.

5. - Accretive operators and nonlinear evolution equations in Banach spaces, 
Proc. Sympos. Pure Math., vol. 18, Part 1, Amer. Math. Soc., Providence, R. I., 1970, pp. 138-161.

6. V. Lakshmikantham and S. Leela, Differential and integral inequalities, vol. I, Academic Press, New York, 1969.

7. R. H. Martin, Jr., A theorem on critical points and global asymptotic stability, J. Math. Anal. Appl. (to appear).

8. - The logarithmic derivative and equations of evolution in a Banach space, J. Math. Soc. Japan 22 (1970), 411-429.

9. G. F. Webb, Nonlinear evolution equations and product integration in Banach spaces, Trans. Amer. Math. Soc. 148 (1970), 273-282.

Georgia Institute of Technology, Atlanta, Georgia 30332 